\title{
Pachydermoperiostosis And An Eyelid Ptosis Associated With Spiky Keratoderma-An Unusual Presentation
}

\author{
Chakraborty $S^{l}$, Sarkar $N S^{2}$, Das $S^{3}$ \\ ${ }^{1} R M O$, Department of Dermatology, NRS Medical \\ College and Hospital, Kolkata; ${ }^{2}$ Assistant Professor, \\ Department of Ophthalmology, NRS Medical \\ College and Hospital, Kolkata; ${ }^{3}$ Associate Professor, \\ Department of Dermatology, NRS Medical College \\ and Hospital, Kolkata
}

\author{
Address for correspondence \\ Dr. Sudip Das
}

Assosciate Professor, Department of Dermatology NRS Medical College and Hospital, Kolkata, India Email: drsudip1966@yahoo.co.in

\section{Citation}

Chakraborty S, Sarkar NS, Das S.

Pachydermoperiostosis and an eyelid ptosis associated with spiky keratoderma-an unusual presentation. NJDVL 2014; 12(1): 48 - 52.

\begin{abstract}
Pachydermoperiostosis, (PDP), a rare hereditary disorder that is characterized by digital clubbing, pachydermia, subperiosteal new bone formation, associated with pain, polyarthritis, cutis verticis gyrata, seborrhoea and hyperhidrosis. It mainly presents due to disfiguring facial features, widening of the limbs and bone and joint pain. It affects male population predominantly and transmitted as an autosomal dominant trait. There are many constant features associated with it as well as rarer features. Increased proliferation of the fibroblast and dysregulation of matrix protein play a central role in the pathogenesis of the disease. They are diagnosed primarily on clinical and radiological grounds. Histopathology plays a supportive role in the diagnosis. Such a case has to be differentiated from several other conditions like acromegaly, neurofibroma, myxedema, primary systemic amyloidosis as well as from other causes of digital clubbing. The case which is described here is a complete form of PDP which is a rare disease reported in Indian context and that too with the a rarer presentation of spiky palmoplanter keratoderma and severe mechanical ptosis leading to severe visual impairment which was corrected after surgical manipulation of the ptosis.
\end{abstract}

Key words: Keratoderma, Pachydermoperiostosis, Ptosis

\section{Introduction}

Pachydermoperiostosis(PDP), is a rare hereditary disorder that is characterized by digital clubbing, pachydermia (thickening of the facial skin and/or scalp), subperiosteal new bone formation, being associated with pain, polyarthritis, cutis verticis gyrata, seborrhea, eyelid ptosis and hyperhidrosis. Though it closely resembles pulmonary hypertrophic osteoarthropathy, in pachydermoperiostosis no causative factors such as pulmonary, cardiac or hepatic disorders have been demonstrated. ${ }^{1-3}$ Existence of the features of periostosis and cutaneous thickening along with the absence of any disease involving any other system of the body suggests PDP. The precise incidence of the disease is unknown ${ }^{1-3}$ but one study has estimated prevalence of $0.16 \%{ }^{4}$

It occurs predominantly and more severely in men with male: female ratio of 7-9:1. The mode of inheritance is autosomal dominant with variable penetrance. It occurs among several members of the family. A familial aggregation of PDP is found in $25 \%$ to $38 \%$ of cases. ${ }^{5}$ Here we describe a case of pachydermoperiostosis with palmoplanter keratoderma which is a very rare association [In south-east Asia].

\section{Case report}

A thirty two year old Muslim male patient presented with recurrent pain of the lower 


\section{Case Report}

extremities for last 15 years, predominantly involving the knee joints along with gradual diminution of vision for last 6 years. This was associated with increased sweating of the hands and feet, gradual thickening of the skin of the face, forearms, palms and soles. There was prominent folding of the skin of the forehead, cheeks, nasolabial folds and increased greasiness skin._This is associated with enlargement of the wrists, elbows, ankles and knees along with the tips of the fingers. Since mid-teens he has been suffering from recurrent increasing knee joint pain along with stiffness. Of late he also complains of mild pain in the hip joints. There is no history of any other medical illness. There is no sensation of warmth in the extremities or effusion of the joints or swelling of the gums. There is no history of similar illness in the family but history of consanguineous marriage among his parents is present.

On clinical examination, the patient was found to have leonine facies with increased furrowing of the forehead folds, broadening of the nasal bridge, high arched palate, cutis verticis gyrata, increased thickening of the hands and feet and spiky punctate keratoderma of the palms and soles (Figure 1). There was sebaceous hyperplasia of the forehead and cheeks, severe digital clubbing and broadening of the wrists and ankles. Tenderness and limited mobility along the knee joints are also observed.

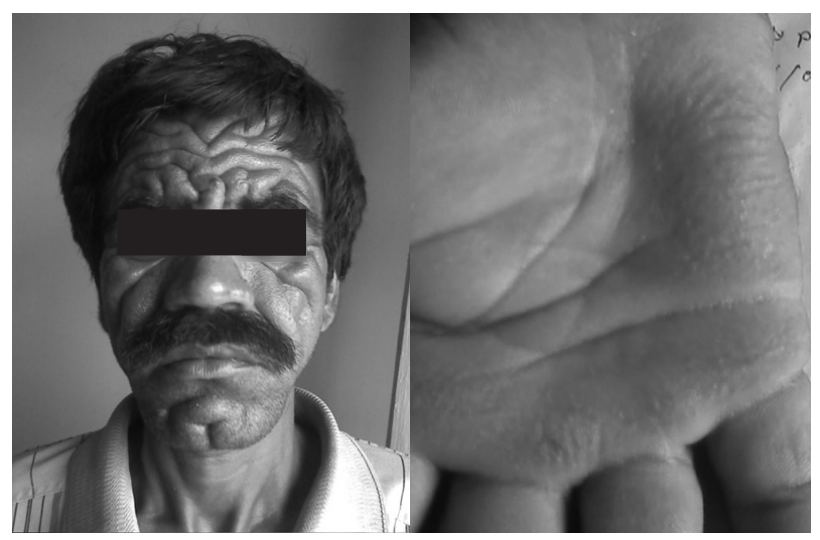

Figure 1: Leonine facies with increased furrowing of the forehead folds, broadening of the nasal bridge, cutis verticis gyrata, increased thickening of the hands and spiky punctuate keratoderma.
Ophthalmological examination revealed thickened eyelids with moderate to severe degree of blepharoptosis, more in the left eye. Margin reflex distance (MRD) is $1.5 \mathrm{~mm}$ (RE) and $0 \mathrm{~mm}$ (LE). Slit-lamp examination showed marked papillary hypertrophy of the tarsal conjunctiva and prominent melanin infiltration at the limbus invading peripheral cornea. Visual acuity $\left(\mathrm{V}_{\mathrm{A}}\right)$ is $6 / 60$ in unaided eyes and $6 / 9 p$ after refraction having myopia with astigmatism against the rule[RE? ? 4.00Dsp. $\mathrm{c}^{-}$? 2.00 Dcyl. at $90^{\circ}$ ?, LE ? ? 6.00Dsp. $\mathrm{c}^{-}$? 1.50 Dcyl. at 100] . The visual field by automated perimetry showed false limitation of the upper field due to blepharoptosis.

General examination was unremarkable except for moderate degree of pallor.

His hemoglobin was $11 \mathrm{~g} / \mathrm{dl}$, total leucocyte count $8900 / \mathrm{mm}^{3}$, erythrocyte sedimentation rate 36 $\mathrm{mm} / \mathrm{h}$. There was normal serum calcium, phosphorus, alkaline phosphatase, uric acid, thyroid hormone and growth hormone profile and normal renal indices. Tests for rheumatoid and antinuclear factors were negative, the VDRL was non-reactive. USG whole abdomen, echocardiography revealed no abnormality. CT scan appears normal.

Radiographic examination showed generalized soft-tissue swelling with digital clubbing, irregular sub-periosteal new bone proliferation with cortical thickening of the distal ends of the long bones mainly involving the distal ends of tibia, fibula, radius, ulna and metacarpals. Radiography of the hands revealed no acrolysis (Figure 2). X-ray chest and the skull were normal. 


\section{Case Report}

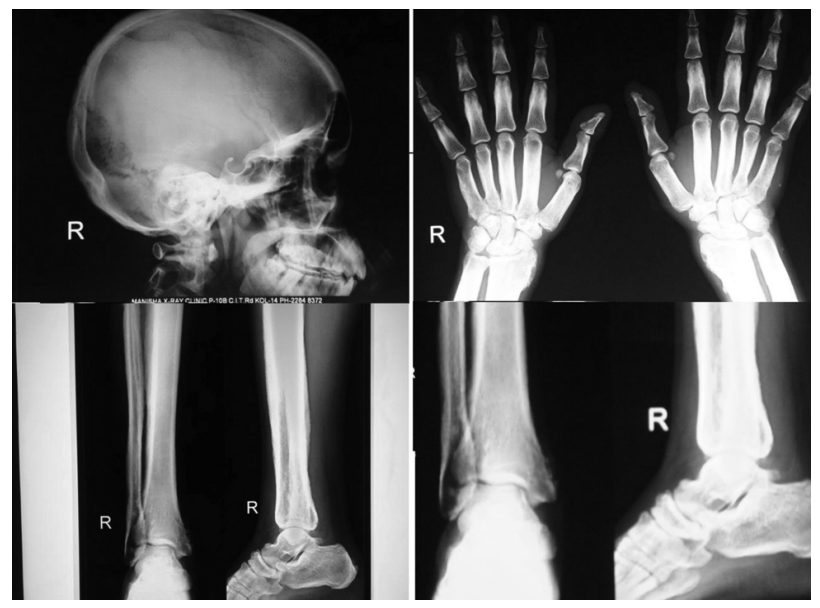

Figure 2: Generalized soft-tissue swelling with digital clubbing, irregular sub-periosteal new bone proliferation with cortical thickening of the distal ends of the long bones mainly involving the distal ends of tibia, fibula, radius, ulna and metacarpals.

Skin biopsy revealed acanthosis with normal granular layer, along with diffuse thickening and packing of collagen in the dermis, thickening of the vessel walls, occlusion of the vascular lumen, sebaceous hyperplasia, dilation of the ducts and the sebaceous glands ( Figure 3).

Patient was treated with topical therapy like salicylic acid and emollients for alleviation of palmoplanter keratoderma. Correction of ptosis surgically by Fassanella -sarvat operation followed by refractive correction leads to considerable improvement in vision.

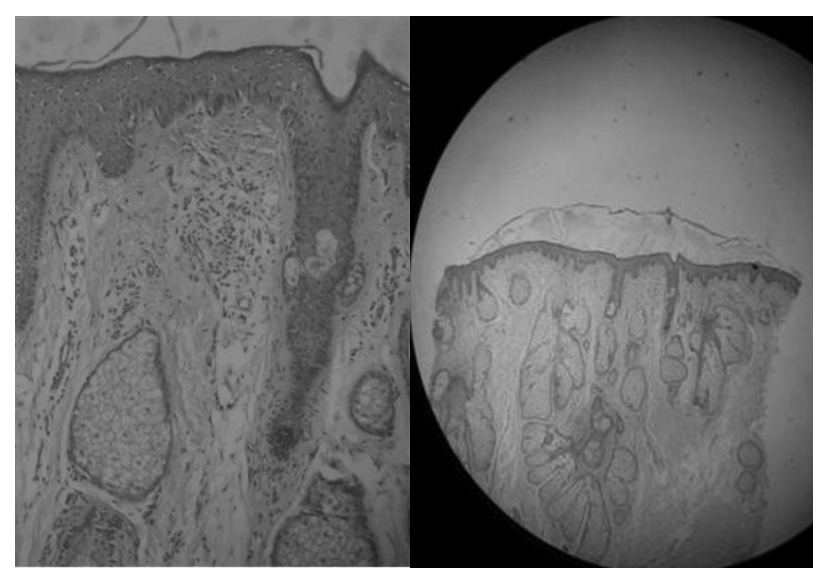

Figure 3: Acanthosis with normal granular layer. Diffuse thickening and packing of collagen in the dermis, thickening of the vessel walls, occlusion of the vascular lumen, sebaceous hyperplasia, dilation of the ducts and the sebaceous glands.

\section{Discussion}

Pachydermoperiostosis or idiopathic hypertrophic osteoarthropathy or Touraine-Solente-Gole syndrome was first described in 1868 by Friedrich in two young brothers. ${ }^{6}$ In 1907, Unna described marked thickening of the skin of the forehead and its resemblance to the sulci and gyri of the brain and called it 'cutis vertices gyrata'? Inherited as an autosomal dominant trait with variable expressivity, one-third of these patients have a positive family history but in our patients this condition is non- familial. ${ }^{8}$

The condition is associated with thickening of the facial skin and scalp and periarticular and subperiosteal periostosis, with consequent enlargement of the hands and feet and joint deformities. ${ }^{3}$ The clinical manifestations of pachydermoperiostosis are somewhat variable with respect to skin and bone changes. The various clinical expressions include the complete form (pachydermia,periostitis, cutis verticis gyrata), the incomplete form (absence of cutis verticis gyrata) and forme fruste (pachydermia with minimal or absent periostitis). ${ }^{8,9}$ The case described here presents as a complete form with cutis verticis gyrata, pachydermia and periostitis.

Little is known of the pathogenesis of pachydermoperiostosis with the exception of the genetic factor. Similarities in clinical manifestations and anatomical findings in pachydermoperiostosis and secondary hypertrophic osteoarthropathy have suggested a common pathogenetic mechanism. ${ }^{10,11}$ Evidence of an increased rate of blood flow through the affected parts has been demonstrated in patients with secondary hypertrophic osteoarthropathy. ${ }^{10,11}$ Increased proliferation of the fibroblast and dysregulation of matrix protein play a central role in the pathogenesis of the disease. ${ }^{3}$

Associated clinical features that have been described commonly include clubbing of digits, arthralgias, seborrheic dermatitis, mechanical 


\section{Case Report}

ptosis of thickened eyelids, periodontal disease and palmoplantar hyperhidrosis. ${ }^{3}$ Our case presented with severe degree of clubbing, seborrhea, palmoplanter hyperhidrosis, mechanical ptosis, arthralgia and arthropathy.

Acro-osteolysis has been reported to be associated, in some patients with this syndrome but we did not corroborate such finding in our patient. ${ }^{11}$

Facial involvement occurs in the form of thickening of the facial skin with prominent folds on the forehead and cheek. A leonine facies is usually a late feature. ${ }^{3}$ Sometimes, the scalp takes on an undulating appearance and shows prominent grooves, being referred as cutis verticis gyrata because of its resemblance to the sulci and gyri of brain. This can also be seen in other conditions like neurofibromatosis, myxedema, cretinism, amyloidosis, acromegaly, etc. as well as in syndromes including Turner's syndrome, Noonan's syndrome, tuberous sclerosis etc. and hence it is not pathognomonic for PDP. But in our patient the association of pachyderma with cutis verticis gyrate along with clubbing and bony changes point the diagnosis towards PDP.

Palmoplanter keratoderma is a rare anecdotal feature reported in very few cases. Our case put up the feature of spiky plamoplanter keratoderma which is a very rare association. ${ }^{13}$ Besides other rare associations like myelodysplasia, gastric hypertrophy, atrophic gastritis and peptic ulcer disease, inflammatory bowel disease, pyoderma gangrenosum, papular mucinosis, SCCs and BCCs had been described. ${ }^{3}$

During the progression of the disease, compressive neuropathy can develop, mainly peripheral as in carpal (or tarsal) tunnel syndrome and rarely femoral head osteonecrosis. Bone marrow insufficiency can also be seen rarely associated with massive endosteal hyperostosis. ${ }^{3}$ The diagnosis is established from clinical and radiological data. It is necessary to exclude secondary forms of hypertrophic osteoarthropathy, which are much more frequent (95\% cases), especially those associated with lung neoplasia and to a lesser degree, with hepatic cirrhosis, cardiomyopathy, chronic obstructive pulmonary disease, bronchiectasia, and some forms of cancer. Differential diagnoses include variants of PDP, secondary hypertrophic osteoarthropathy, thyroid acropachy, acromegaly, van Buchem's disease (in which there is absence of clubbing and skin changes), diaphyseal dysplasia (endosteal and periosteal proliferation), and syphilitic periostosis. Variants of PDP include Rosenfeld-Kloepfer syndrome (characterized by enlargement of the jaws, especially mandible, and of the hands and feet, nose, lips, tongue, and forehead, along with cutis vertices gyrata and corneal leukoma); Currarino idiopathic osteoarthropathy (an incomplete form of PDP seen in children and adolescents and characterized by the presence of eczema and sutural diastases); and a localized form with only the radiographic features of PDP in the lower extremities.

To summarise the case we describe here is a complete form of PDP associated with rare feature of spiky palmoplanter keratoderma along with marked visual impairment secondary to mechanical ptosis associated with astigmatism. PDP is a rare disorder with varied manifestations and associations. The case which is described here is a complete form of PDP which is a rare disease reported in Indian context and that too with the a rarer presentation of spiky palmoplanter keratoderma and severe mechanical ptosis leading to severe visual impairment which was corrected after surgical manipulation of the ptosis. Treatment is limited to NSAID, steroid, or colchicine therapy to alleviate arthralgias and retinoids for the dermal changes. Surgical treatment is limited to plastic surgery for cosmetic indications or correction of associated deformities. Early diagnosis helps in reducing the morbidity and in prognostication. 
Case Report

\section{References}

1. Kowalewski M, Urban M, Górska A. Familial occurrence of primary hypertrophic osteoarthropathy: A case report. Med Sci Monit 1997; 3: 229-34.

2. Mattuci-Cerinic M, Lotti T, Jajic I, Pignole A, Bussani C, Cagnoni M.The clinical spectrum of pachydermoperiostosis (primary hypertrophic osteoarthropathy). Medicine 1991; 70: 208-14.

3. Auger M, Stavrianeas N. Pachydermoperiostosis. Orphanet Enciclopedia 2004: 1-8.

4. Jajic I, Jajic Z. Prevalence of primary hypertrophic osteoarthropathy in selected population. Clin Ex Rheum 1992; 10: 73.

5. Kowalewski M, Urban M, Górska A. Familial occurrence of primary hypertrophic osteoarthropathy: A case report. Med Sci Monit 1997; 3: 229-34.

6. Carcassi U. History of hypertrophic osteoarthropathy (HOA). Clin Exp Rheumatol 1992; 10: 3-7.
7. Unna PG. Cutis Verticis Gyrata: Monatsschr Praktische. Deramatol 1907; 45: 227.

8. Lavin MM, Pineda C, Valdez T, Cajigas JC, Weisman M, Gerber N et al. Primary hypertrophic osteoarthropathy. Semin Arthritis Rheum 1988; 17: 156-62.

9. Harbison J B, Nice C M Jr. Familial Pachydermoperiostitis presenting as an acromegaly like syndrome. AJR 1971; 112: 532.

10. Ursing B. Pachydermoperiostosis. Acta Med Scand 1970; 188: 57.

11. Joseph B, Chacko V. Acro-osteolysis with Hypertrophic Pulmonary Osteoarthropathy and Pachydermoperiostosis. Radiology; 1985: 154: 343-4.

12. Jajic Z, Jajic I. Radiological changes of short and flat bones in primary hypertrophic osteoarthropathy. Ann Rheum Dis 1998; 57: 747-50.

13 . Barraud-Klenovsek MM, Lubb J, Burg G. Primary digital clubbing associated with palmoplanter keratoderma. Dermatology 1997; 194: 302-5. 